\title{
Ultrastructural changes in muscle cells of patients with collagen Vl-related myopathies
}

\author{
Francesca Tagliavini ${ }^{1}$ \\ Francesca Sardone ${ }^{1}$ \\ Stefano Squarzoni ${ }^{1}$ \\ Nadir Mario Maraldi ${ }^{1}$ \\ Luciano Merlini² \\ Cesare Faldini ${ }^{3}$ \\ Patrizia Sabatelli ${ }^{1}$
}

1 SC Laboratory of Muscoloskeletal Cell Biology, IOR, Bologna, Italy; CNR- National Research Council of Italy, Institute of Molecular Genetics, Bologna, Italy

2 SC Laboratory of Muscoloskeletal Cell Biology, IOR, Bologna, Italy

3 University of Bologna, Rizzoli Orthopaedic Institute, Bologna, Italy

Corresponding author:

Francesca Tagliavini

SC Laboratory of Muscoloskeletal Cell Biology, IOR

Via di Barbiano, 1/10

40136 Bologna, Italy

E-mail: tagliavini.fra@gmail.com

\section{Summary}

Collagen VI is an extracellular matrix protein expressed in several tissues including skeletal muscle. Mutations in COL6A genes cause Bethlem Myopathy (BM), Ullrich Congenital Muscular Dystrophy (UCMD) and Myosclerosis Myopathy (MM). Collagen VI deficiency causes increased opening of the mitochondrial permeability transition pore (mPTP), leading to ultrastructural and functional alterations of mitochondria, amplified by impairment of autophagy. Here we report for the first time ultrastructural studies on muscle biopsies from BM and UCMD patients, showing swollen mitochondria with hypodense matrix, disorganized cristae and paracrystalline inclusions, associated with dilated sarcoplasmic reticulum and apoptotic changes. These data were supported by scanning electron microscopy analysis on BM and UCMD cultured cells, showing alterations of the mitochondrial network. Morphometric analysis also revealed a reduced short axis and depicted swelling in about $3 \%$ of mitochondria. These data demonstrate that mitochondrial defects underlie the pathogenetic mechanism in muscle tissue of patients affected by collagen VI myopathies.
KEY WORDS: collagen VI, mitochondria, MPTP, scanning electron microscopy back-scattered imaging, transmission electron microscopy.

\section{Introduction}

Collagen VI (ColVI) is a protein widely expressed in the extracellular matrix (ECM) of most connective tissues where it contributes to the maintenance of tissues integrity and function ${ }^{1}$. It forms a microfibrillar network in several organs including skeletal muscle where it is mainly localized just outside the basement membrane ${ }^{2}$. ColVI is composed by three chains (alpha1, alpha2, alpha3), encoded by different genes (COL6A1, COL6A2 and COL6A3, respectively), which associate inside the cell in a stoichiometric ratio to form a triple helical monomer which assembles in dimers and tetramers stabilized by disulfide bonds ${ }^{3}$. After secretion, tetramers associate end-to-end, forming typical $100 \mathrm{~nm}$ periodic beaded microfibrils by interactions of their non-collagenous domains. Recently, three novel ColVI chains (alpha4, alpha5, and alpha6) were discovered, which resemble alpha3 but have a more restricted tissue distribution ${ }^{4}$. Novel ColVI chains may replace alpha3, probably forming alpha 1-2-4, alpha 1-2-5, or alpha 1-2-6 heterotrimers ${ }^{4}$. Mutations in COL6A1, COL6A2 and COL6A3 genes cause a group of inherited muscular dystrophies: Ullrich Congenital Muscular Dystrophy (UCMD), Bethlem Myopathy (BM) and Myosclerosis Myopathy (MM). UCMD is a severe disorder characterized by congenital muscle weakness with axial and proximal joint contractures and coexisting distal joint hypermobility 5 . BM is an autosomal dominant or recessive disorder characterized by slowly progressive axial and proximal muscle weakness with finger flexion contractures ${ }^{6}$. $\mathrm{MM}$ is a recessive disorder characterized by progressive all-joint contractures ${ }^{7}$.

Collagen VI myopathies share a mitochondrial dysfunction due to the deregulation of the permeability transition pore (mPTP) ${ }^{8}$. In vitro studies on $\mathrm{BM}$ and UCMD mitochondria show an abnormal depolarization unmasked by the employment of oligomycin, an ATP synthase inhibitor ${ }^{9}$. The identification of the pathogenic mechanism of ColVI myopathies paved the way to several treatments to rescue the mitochondrial defect, using compounds or genetic manipulations. The normalizing effect of cyclosporin A (CsA) on mPTP pore was demonstrated in vitro and ex vivo conditions ${ }^{9}$, then employed in a pilot clinical trial ${ }^{10}$. CsA in fact inhibits the MPTP through the binding and the enzymatic block of cyclophilin (CyP) D, a mitochondrial matrix 
peptidyl-prolylcis-transisomerase (PPlase) ${ }^{11}$. Moreover, Debio 025, a non-immunosuppressive derivative of CsA successfully rescued the mPTP function ${ }^{12}$. A different approach was adopted by inactivating the Ppif gene encoding cyclophilin D in the Col6a1 -/mouse model, confirming the pathogenic role of mPTP opening ${ }^{13}$. A strong mitochondrial involvement has been found also in the Col6a1 -/- mouse model which showed a defective autophagy ${ }^{14}$. The impaired removal of defective mitochondria amplifies the defect, which is worsened by excessive oxidative stress and $\mathrm{Ca}^{2+}$ accumulation in the mitochondria. The autophagy defect correction by a low protein diet demonstrated a morphological mitochondrial rescue in terms of size and decrease of swelling ${ }^{14}$.

Additional evidences for the mitochondrial involvement in the pathogenesis of collagen VI-related myopathies derived from zebrafish animal model, genetically manipulated on col6a1 gene to reproduce both the mild phenotype (BM-like) and the strong phenotype (UCMD-like) ${ }^{15}$. This resulted a good model to study because of the mPTP structural and functional homology between zebrafish and mammals ${ }^{16}$. Treatment with CsA was in fact effective on both phenotypes, restoring mitochondrial abnormalities and decreasing the apoptotic rate, together with a strong improvement of motor function ${ }^{15}$.

Here we report for the first time ultrastructural studies on muscle biopsies from BM and UCMD patients, presenting swollen mitochondria with hypodense matrix, disorganized cristae and paracrystalline inclusions associated with dilated sarcoplasmic reticulum and apoptotic changes. The same mitochondrial alterations are maintained in BM and UCMD cell cultures, which can be regarded as a reliable model for the study of these diseases.

\section{Materials and Methods}

\section{Patients}

Skeletal muscle biopsies from three healthy subjects, two UCMD patients, two BM patients and one MM patient were collected; samples were frozen in prechilled isopentane and stored in liquid nitrogen. Patients were diagnosed by genetic, biochemical and immunohistochemical analysis: UCMD-1, UCMD-2 and BM patients were described by Merlini et al. ${ }^{17}$ (patient 1 , patient 3 and patient 5; respectively); MM patient was described by Merlini et al. ${ }^{7}$. All participants provided written informed consent, and approval was obtained from the Ethics Committee of the Rizzoli Orthopaedic Institute (Bologna, Italy).

\section{Primary muscle-derived cell cultures}

Primary cell cultures derived from muscle biopsies of control, BM patient and UCMD patients were established and maintained in Dulbecco's modified Eagle's medium (DMEM) plus 20\% fetal calf serum (FCS) and antibiotics (penicillin, streptomycin and amphotericin B).

\section{Transmission electron microscopy}

Muscle biopsies were processed for electron microscopy analysis. Muscle tissue was fixed with $2.5 \%$ glutaraldehyde- $0.1 \mathrm{M}$ cacodylate buffer $\mathrm{pH} 7.4$ for $3 \mathrm{~h}$ at $4{ }^{\circ} \mathrm{C}$, post-fixed with $1 \%$ osmium tetroxide (OsO4) in cacodylate buffer for $2 \mathrm{~h}$, and dehydrated in an ethanol series, infiltrated with propylene oxide and embedded in Epon 812 resin.

Muscle-derived cells were grown in DMEM supplemented with $20 \%$ FCS until confluence, fixed with $2.5 \%$ glutaraldehyde in cacodylate buffer $0.1 \mathrm{M}, \mathrm{pH}$ 7.2 , and post-fixed with $1 \%$ osmium tetroxide in cacodylate buffer $0.1 \mathrm{M}, \mathrm{pH}$ 7.3. After detaching with propylene oxide, the cells were centrifuged and embedded in Epon 812. Utrathin sections were stained with uranyl acetate and lead citrate and observed with a Philips EM400 operating at $100 \mathrm{kV}$.

\section{Scanning electron microscopy back-scattered imaging}

$\mathrm{Cu}-\mathrm{Pb}$ staining solution was prepared by mixing $20 \mathrm{ml}$ of $4.6 \%$ trisodium citrate, $2 \mathrm{ml}$ of $33 \%$ lead nitrate, and $0.5 \mathrm{ml}$ of $10 \%$ copper sulfate $(\mathrm{pH} 9)$, and used undiluted. Cells were fixed with $2.5 \%$ glutaraldehyde- $0.1 \mathrm{M}$ cacodylate buffer $\mathrm{pH} 7.3$ for $1 \mathrm{~h}$ at $4^{\circ} \mathrm{C}$. After washing with $0.1 \mathrm{M}$ cacodylate buffer $\mathrm{pH} 7.3$, the coverslips were brought to room temperature, washed three times with distilled water and incubated with $\mathrm{Cu}-\mathrm{Pb}$ solution at $4^{\circ} \mathrm{C}$ overnight. The samples were then brought to room temperature, washed three times with distilled water and post-fixed with $1 \%$ osmium tetroxide in $0.1 \mathrm{M}$ cacodylate buffer for $1 \mathrm{~h}$ at room temperature. After rinsing with $0.1 \mathrm{M}$ cacodylate buffer and then with distilled water, the samples were dehydrated in an ethanol series and critical point dried. The coverslips carrying cells were then mounted on aluminum stubs with silver adhesive paint, coated with spectrographic carbon by vacuum evaporation in an Ewards E 306 apparatus, and observed at $0^{\circ}$ tilt angle with a Cambridge Stereoscan 200 scanning electron microscope equipped with an Oxford Tetra solid state BSE detector. Acceleration voltage was set at $30 \mathrm{kV}$ and WD at $15 \mathrm{~mm}$.

\section{Immunofluorescence analysis}

For immunofluorescence analysis in vitro, muscle cells were grown onto coverslips, fixed with cold methanol, washed with PBS and incubated with rabbit anti-desmin (Monosan Technology) and Mito Tracker Red (Life Technologies). Nuclei were counterstained with DAPI. Samples were mounted with an anti-fading reagent (Molecular Probes) and observed with a Nikon epifluorescence microscope. 


\section{Results}

By transmission electron microscopy, we investigated the ultrastructural alterations in muscle fibers of collagen VI-related myopathies patients. In UCMD patients muscle fibers we noticed a strong enlargement of sarcoplasmic reticulum associated with mitochondria abnormalities including swelling and cristae with a tubular arrangement. Mitochondrial alterations were also detected in muscle fibers of $\mathrm{MM}$ and $\mathrm{BM}$ patients. The unusual presence of osmiophilic bodies and paracrystalline inclusions was found in several non necrotic muscle fibers, as indicated by the normal organization of myofibrils (Fig. 1A). Interestingly, muscle fibers of UCMD patients showed also the presence of nuclear changes consistent with apoptosis, as indicated by the peripheral chromatin condensation and irregular shape (Fig. 1B).

To better characterize the mitochondrial network, muscle cell cultures were studied by using the mitochondrion-selective probe MitoTracker ${ }^{18}$. MitoTracker is a fluorescent dye that stains mitochondria in live cells and its accumulation is dependent upon membrane potential. Consistent with a latent mitochondrial dysfunction ${ }^{9}$, UCMD and BM muscle cells, identified by anti-desmin antibody, displayed mitochondrial changes consistent with fragmentation as indicated by the presence of a perinuclear punctuate pattern with respect to the elongated and well developed network of control cells (Fig. 2A). Back scattered imaging of cultured cells allowed to clearly monitor both organization and extension of the mitochondrial reticulum by copper-lead salt solution which specifically stains the mitochondria membranes ${ }^{19}$. BM and UCMD patient cells showed reduced mitochondrial branching, increased mitochondria fragmentation and swelling, and irregular thickness, with respect to the control which displayed a regular long and branched mitochondrial reticulum (Fig. 2B). A morphological analysis of the mitochondrial reticulum was also conducted on resin embedded samples. In agreement with the scanning electron microscopy analysis, UCMD and BM mitochondria showed an irregular shape and changes consistent with mitochondrial swelling (Fig. $3 A)$. These features were also confirmed by morphometric analysis (Fig. 3B); in fact, UCMD and BM patients displayed a high percentage of smaller mitochondria as compared to control (mitochondria with short axis $<200 \mathrm{~nm}$ : $29.3 \%$ in control, $46.55 \%$ in $\mathrm{BM}$ patient and $61.21 \%$ in UCMD patient) and also a significant increase of mitochondria with short axis $>600 \mathrm{~nm}$ : $0,86 \%$
A
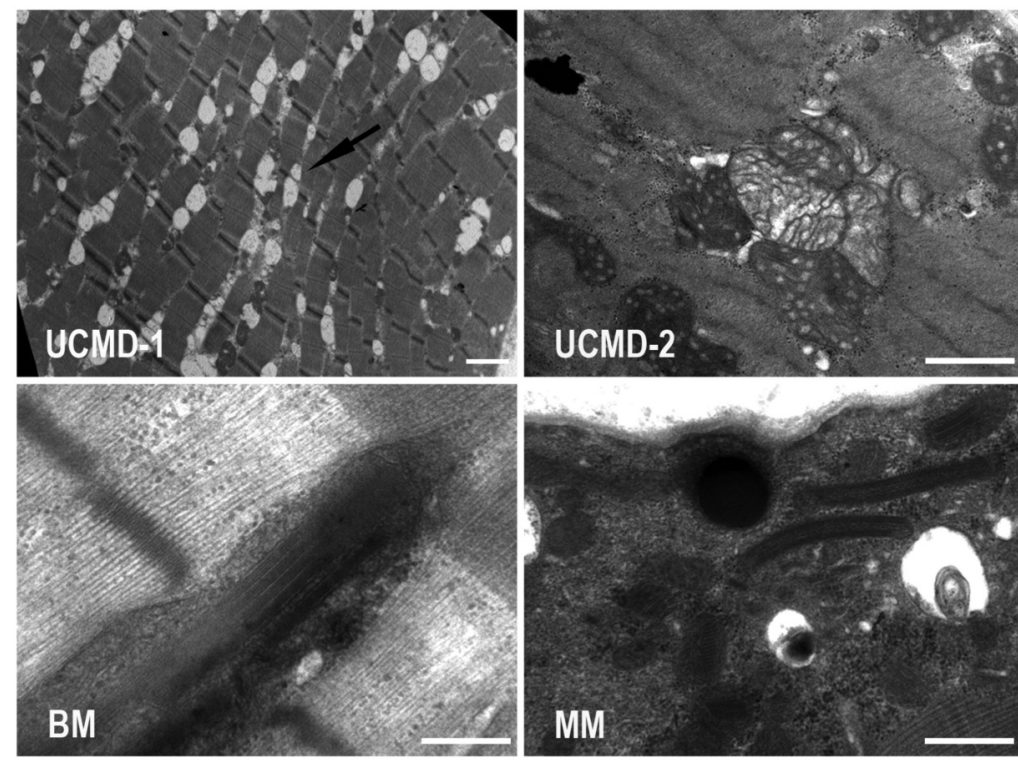

B

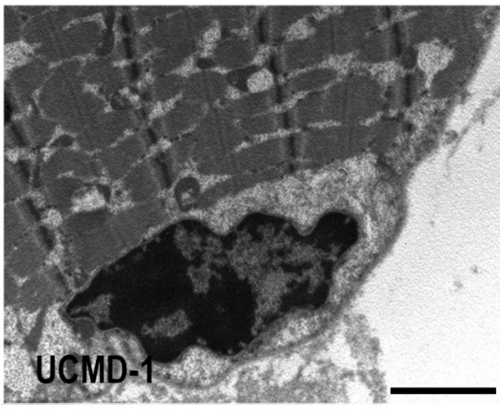

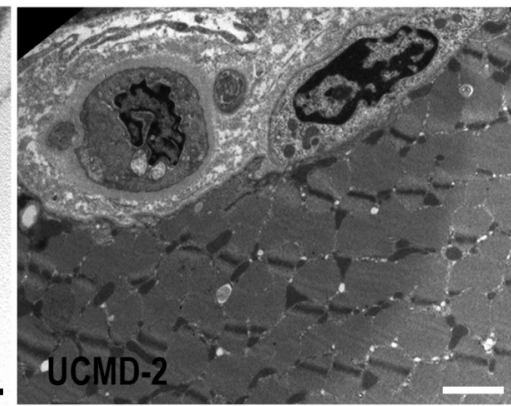

Figure 1. (A) Transmission electron microscopy analysis of Eponembedded muscular biopsies from UCMD, BM and MM patients. All samples display ultrastructural alterations: sarcoplasmic reticulum enlargement associated with mitochondria abnormalities as well as osmiophilic bodies and paracrystalline inclusions. Scale bar, $0.5 \mu \mathrm{m}$. (B) A muscle fiber of a UCMD patient showing nuclear changes consistent with apoptosis, such as an irregular shape and chromatin condensation. Scale bar, $0.5 \mu \mathrm{m}$. 

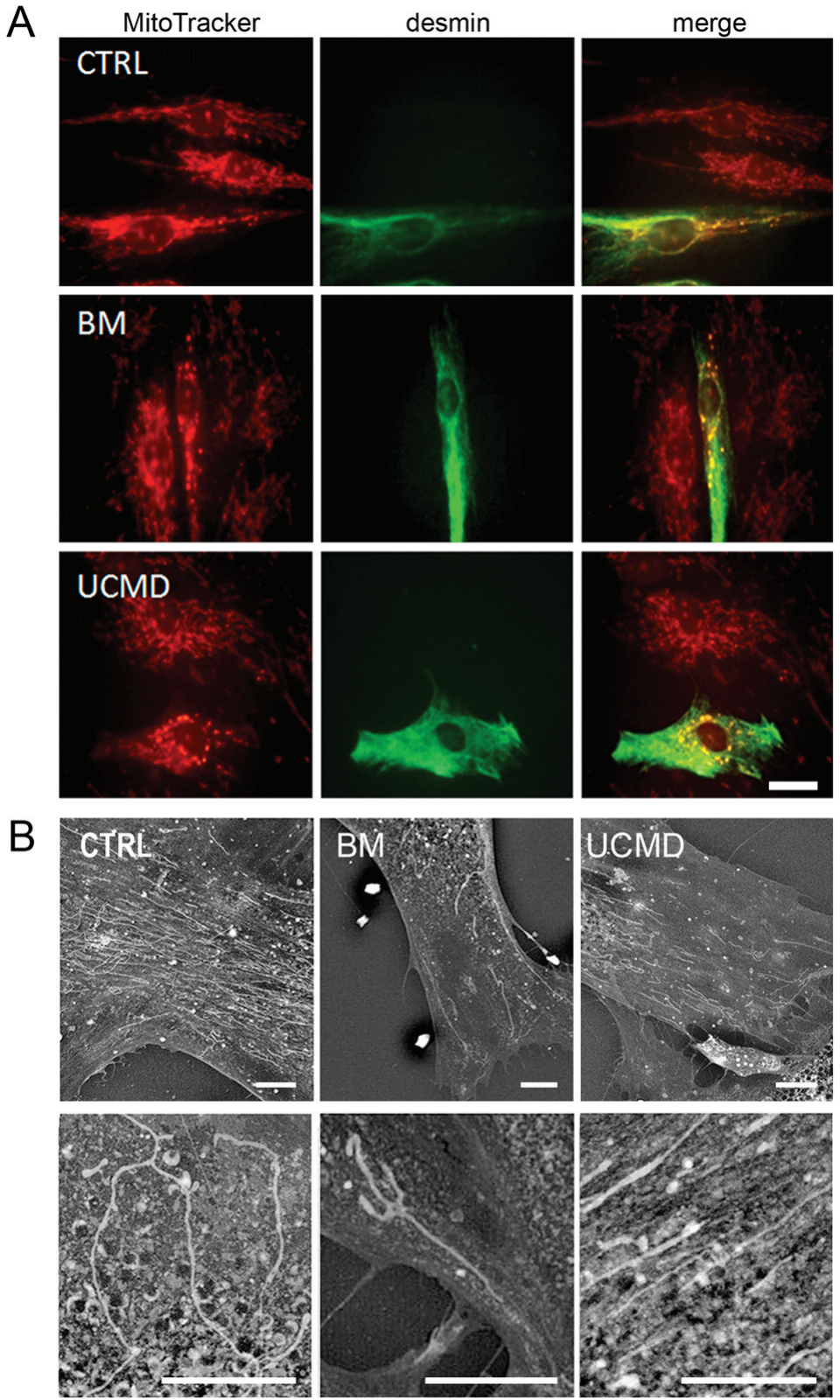

Figure 2. (A) Mitotracker red labelling on control, UCMD and BM muscle cells, identified by anti-desmin labelling (green). In these representative images the mitochondrial network appears fragmented and discontinuous in both patients as compared to that of control cells. Scale bar, $20 \mu \mathrm{m}$. (B) This pattern is confirmed by back scattered imaging of muscle-derived cell cultures from BM and UCMD patients. Both patients display a less developed mitochondrial network compared to the long and branched one in the control. Scale bar, 25 $\mu \mathrm{m}$. in control, $2.58 \%$ in BM and UCMD patients, which can be interpreted as swelling events (Fig. 3B).

\section{Discussion}

Mitochondria have been recently recognized as new leading actor in collagen VI myopathies. The first observation came from Irwin et al. ${ }^{20}$ which demonstrated the existence of a latent mitochondrial dysfunction in a knockout mouse model (Col6a1-/-). The mitochondrial defect was also observed in myofibers isolated from muscle of Col6a1-/- mice after the addition of oligomycin, thus inhibiting ATP synthase and dropping the mitochondrial membrane potential ${ }^{20}$.
Until now, the alterations responsible of the pathogenic mechanism of collagenVI myopathies have been described in animal models (Col61-/- knockout mouse and col6 mutant zebrafish) and in patient cell cultures. Here we report for the first time that mitochondrial abnormalities are also present in mature muscle fibers of UCMD, BM and MM patients, indicating that mitochondrial defect underlie the pathogenetic mechanism in muscle tissue of patients affected by collagen VI myopathies. Morphometric analysis and scanning electron microscopy of mitochondrial network in UCMD and BM cultured cells suggests alterations of mitochondrial reticulum with a tendency to swelling and fragmentation in collagen VI-deficient cells. 

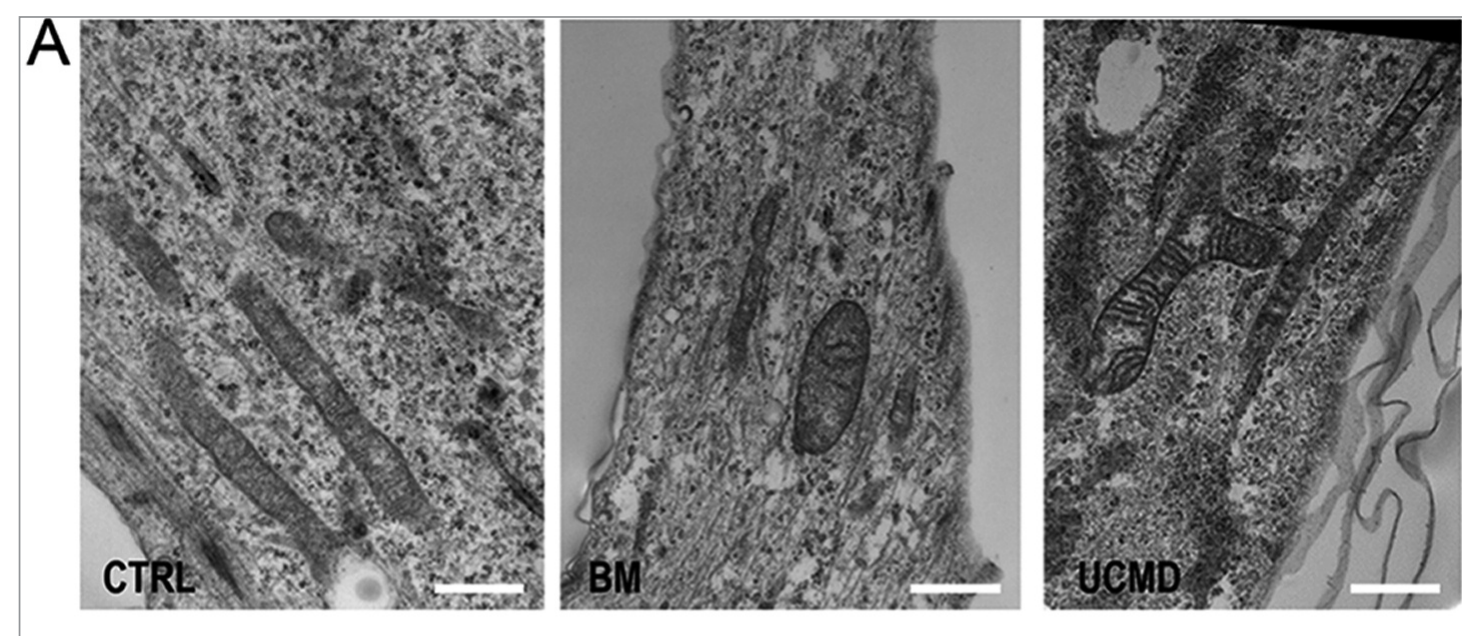

B

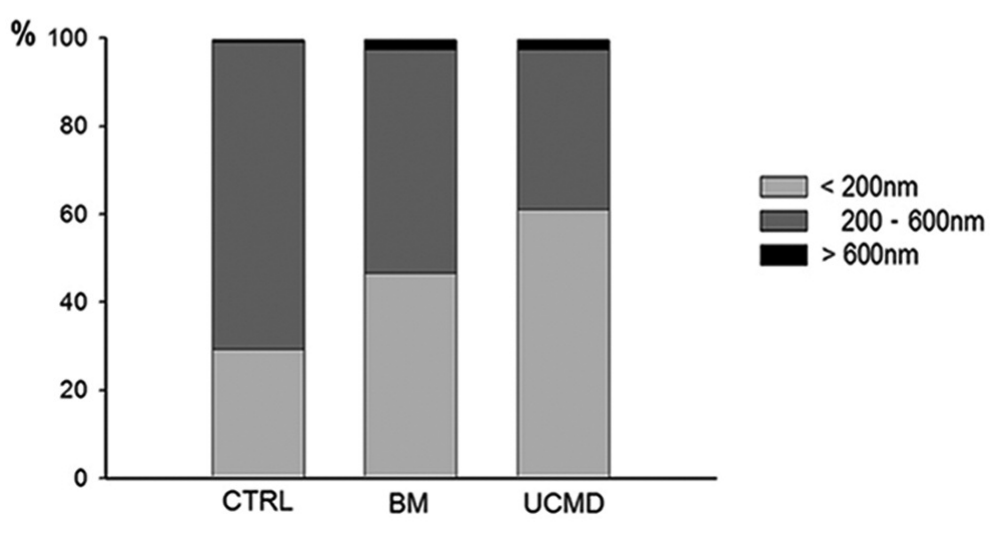

Figure 3. (A) Transmission electron microscopy analysis of Epon-embedded cell cultures from control, BM and UCMD patients. Patients' cells show morphological alteration consisting in irregularly shaped and swollen mitochondria. Scale bar, 0.8 $\mu \mathrm{m}$. (B) Morphometric analysis of the mitochondrial short axis. UCMD and BM patients display an higher percentage of smaller mitochondria as compared to control (mitochondria with short axis measuring less than 200nm: 29.3 in control, 46.55 in BM patient and 61.21 in UCMD patient) and also a clear increase of mitochondria percentage with short axis measuring more than 600nm (0,86 in control, 2.58 in BM and UCMD patients) which can be interpreted as swelling events.

Altered mitochondrial morphology associated with mPTP dysfunction have been reported in cultured muscular cells of patients affected by collagen VI myopathies ${ }^{9}$. Muscular dystrophies linked to collagen $\mathrm{VI}$ deficiency show dysfunctional mitochondria and spontaneous apoptosis, leading to myofiber degeneration.

The MPTP latent dysfunction was observed moreover both in muscle cells and in muscle fibroblasts from UCMD and BM patients ${ }^{21}$, confirming that mitochondrial alteration is a key feature of collagen VI-related diseases. Protracted mPTP opening can disable respiration, producing toxic oxidative species and mediating leakage of proteins into the cytoplasm that can activate apoptosis or necrosis 22 . Interestingly, we recently reported that DMD muscle cells and melanocytes displayed the oligomycin-induced mitochondrial depolarization ${ }^{23}$.

The autophagy defect due to collagen VI deficiency in the Col6a1 -/- mouse model and BM and UCMD pa- tients leads furthermore to the accumulation of altered mitochondria ${ }^{14}$. Defect of the autophagic system cause a strong impairment in the degradative mechanism of abnormal organelles ${ }^{14}$.

The data here reported confirm the importance of mitochondria in the pathogenesis of ColVI muscular dystrophies. The collagen VI-mitochondria connection requires the understanding of the mechanisms by which mitochondria sense alterations in the ECM. The data here reported, which indicate that in tissues and cell cultures from ColVI-affected patients mitochondria undergo not only swelling, possibly due to the $\mathrm{Ca}^{2+}$ overload, but also fragmentation of their network, suggest that additional players could be involved. Further studies should confirm the hypothesis that the regulation of mitochondrial distribution and that of the cytoskeletal three-dimensional structure, which affect the sensitivity of cells to apoptotic stimuli ${ }^{24}$, depend on the small GTPases that modulate the fusion-fission machinery 25 . 


\section{Acknowledgements}

This work was supported by TELETHON Italy (GUP11007 to LM, PS and NMM and GGP11082 to NMM and PS) by FIRB 2010 RBAP10KCNS and "5 per mille" 2010 Rizzoli Orthopaedic Institute.

\section{References}

1. Keene DR, Engvall E, Glanville RW. Ultrastructure of type $\mathrm{VI}$ collagen in human skin and cartilage suggests an anchoring function for this filamentous network. The Journal of cell biology 1988; 107(5):1995-2006.

2. Kuo HJ, Maslen CL, Keene DR, Glanville RW. Type VI collagen anchors endothelial basement membranes by interacting with type IV collagen. J Biol Chem 1997; 272(42):26522-26529.

3. Engvall E, Hessle H, Klier G. Molecular assembly, secretion, and matrix deposition of type $\mathrm{VI}$ collagen. The Journal of cell biology 1986; 102(3):703-10.

4. Gara SK, Grumati P, Urciuolo A, et al. Three novel collagen $\mathrm{VI}$ chains with high homology to the alpha3 chain. J Biol Chem 2008; 283(16):10658-10670.

5. Bertini E, Pepe G. Collagen type VI and related disorders: Bethlem myopathy and Ullrich scleroatonic muscular dystrophy. Eur J Paediatr Neurol 2002; 6(4):193-198.

6. Merlini L, Morandi L, Granata C, Ballestrazzi A. Bethlem myopathy: early-onset benign autosomal dominant myopathy with contractures. Description of two new families. Neuromuscul Disord 1994; 4(5-6):503-511.

7. Merlini L, Martoni E, Grumati $P$, et al. Autosomal recessive myosclerosis myopathy is a collagen VI disorder. Neurology 2008; 71(16):1245-1253.

8. Bernardi P, Krauskopf A, Basso E, et al. The mitochondrial permeability transition from in vitro artifact to disease target. FEBS J 2006; 273(10):2077-2099.

9. Angelin A, Tiepolo T, Sabatelli P, et al. Mitochondrial dysfunction in the pathogenesis of Ullrich congenital muscular dystrophy and prospective therapy with cyclosporins. Proc Natl Acad Sci U S A 2007; 104(3):991-996.

10. Merlini L, Bernardi P. Therapy of collagen VI-related myopathies (Bethlem and Ullrich). Neurotherapeutics 2008; (4):613-618.

11. Fischer G, Wittmann-Liebold B, Lang K, Kiefhaber T, Schmid FX. Cyclophilin and peptidyl-prolyl cis-trans isomerase are probably identical proteins. Nature 1989; 337(6206):476-478.
12. Tiepolo T, Angelin A, Palma E, et al. The cyclophilin inhibitor Debio 025 normalizes mitochondrial function, muscle apoptosis and ultrastructural defects in Col6a1-/- myopathic mice. Br J Pharmacol 2009; 157(6):1045-1052.

13. Palma E, Tiepolo T, Angelin A, et al. Genetic ablation of cyclophilin $\mathrm{D}$ rescues mitochondrial defects and prevents muscle apoptosis in collagen VI myopathic mice. Human molecular genetics 2009; 18(11):2024-2031.

14. Grumati $P$, Coletto $L$, Sabatelli $P$, et al. Autophagy is defective in collagen VI muscular dystrophies, and its reactivation rescues myofiber degeneration. Nat Med 2010; 16(11):131320.

15. Telfer WR, Busta AS, Bonnemann CG, Feldman EL, Dowling JJ. Zebrafish models of collagen VI-related myopathies. Human molecular genetics 2010; 19(12):2433-2444.

16. Azzolin L, Basso E, Argenton F, Bernardi P. Mitochondrial $\mathrm{Ca} 2+$ transport and permeability transition in zebrafish (Danio rerio). Biochim Biophys Acta 2010; 1797(11):17751779.

17. Merlini L, Sabatelli P, Armaroli A, et al. Cyclosporine A in Ullrich congenital muscular dystrophy: long-term results. Oxid Med Cell Longev (2011); ID139194.

18. Chazotte B. Labeling mitochondria with MitoTracker dyes. Cold Spring Harb Protoc 2011; 2011(8):990-992.

19. Thiery G. Colorations permettant l'étude des coupes épaisses, leur intéret en miscoscopie électronique. J Miscosc 1973; 17:101A.

20. Irwin WA, Bergamin N, Sabatelli $P$, et al. Mitochondrial dysfunction and apoptosis in myopathic mice with collagen $\mathrm{VI}$ deficiency. Nature genetics 2003; 35(4):367-371.

21. Sabatelli P, Palma E, Angelin A, et al. Critical evaluation of the use of cell cultures for inclusion in clinical trials of patients affected by collagen VI myopathies. Journal of cellular physiology 2012; 227(7):2927-2935.

22. Bernardi P, Bonaldo P. Mitochondrial dysfunction and defective autophagy in the pathogenesis of collagen VI muscular dystrophies. Cold Spring Harbor perspectives in biology 2013; 5(5):a011387.

23. Pellegrini C, Zulian A, Gualandi F, et al. Melanocytes-a novel tool to study mitochondrial dysfunction in Duchenne muscular dystrophy. Journal of cellular physiology 2013; 228(6):1323-1331.

24. Frank S, Gaume B, Bergmann-Leitner ES, et al. The role of dynamin-related protein 1 , a mediator of mitochondrial fission, in apoptosis. Developmental cell 2001; 1(4):515-525.

25. McNiven MA, Cao H, Pitts KR, Yoon Y. The dynamin family of mechanoenzymes: pinching in new places. Trends in biochemical sciences 2000; 25(3):115-120. 